\title{
Clausius relation for Active Particles
}

\section{Maggi, A. Puglisi, U. Marini Bettolo Marconi}

A. Puglisi and U. Marini Bettolo Marconi, Entropy 19, 356 (2017)

U. Marini Bettolo Marconi, A.Puglisi, C.Maggi, Scientific Reports 7, 46496 (2017) 


\section{Clausius relation in}

\section{macroscopic thermodynamics}

$\delta Q$ is the heat exchanged with the thermostat during a transformation

$$
\begin{gathered}
\delta \Sigma=d S-\frac{\delta Q}{T} \geq 0 \quad \text { is the entropy production } \\
\oint \frac{\delta Q(t)}{T(t)} \leq 0 \quad \begin{array}{l}
\text { is the Clausius relation for a } \\
\text { cyclical transformation }
\end{array}
\end{gathered}
$$




\section{Mesoscopic description}

$$
\left\{\omega\left(t^{\prime}\right)\right\}_{0}^{t} \text { is a stochastic trajectory }
$$

$$
\begin{gathered}
\ln \frac{\operatorname{prob}\left[\left\{\omega\left(t^{\prime}\right)\right\}_{0}^{t}\right]}{\operatorname{prob}\left[\left\{\bar{\omega}\left(t-t^{\prime}\right)\right\}_{0}^{t}\right]}=\ln \frac{p[\omega(0)]}{p[\overline{\omega(t)}]}+\ln \frac{\operatorname{prob}\left[\left\{\omega\left(t^{\prime}\right)\right\}_{0}^{t} \mid \omega(0)\right]}{\operatorname{prob}\left[\left\{\bar{\omega}\left(t-t^{\prime}\right)\right\}_{0}^{t} \mid \bar{\omega}(t)\right]} \\
\int_{0}^{t} \delta \sigma\left(t^{\prime}\right)=\int_{0}^{t} d s+\int_{0}^{t} \delta s_{m} \\
\delta s_{m}=-\delta q / T
\end{gathered}
$$

$\underline{\text { Steady state averages }}$

$$
\delta \Sigma=\langle\delta \sigma\rangle \geq 0 \quad d S=\langle d s\rangle \quad\left\langle\delta s_{m}\right\rangle=-\delta Q / T \geq 0
$$




\section{A simple example}

$$
\begin{gathered}
d x(t)=u(t) d t \\
m d u(t)=-\gamma u(t) d t+\sqrt{2 \gamma T} d W(t)-\phi^{\prime}[x(t)] d t+f_{n c}(t) d t \\
e=\frac{m u^{2}}{2}+\phi(x) \\
\delta w=u f_{n c} d t \quad \delta q=d e-\delta w=u \circ[-\gamma u d t+\sqrt{2 \gamma T} d W(t)] \\
\delta \sigma=d s-\frac{u \circ[-\gamma u d t+\sqrt{2 \gamma T} d W]}{T}=d s-\frac{\delta q}{T}
\end{gathered}
$$




\section{Active particles}

\section{Self-propulsion}

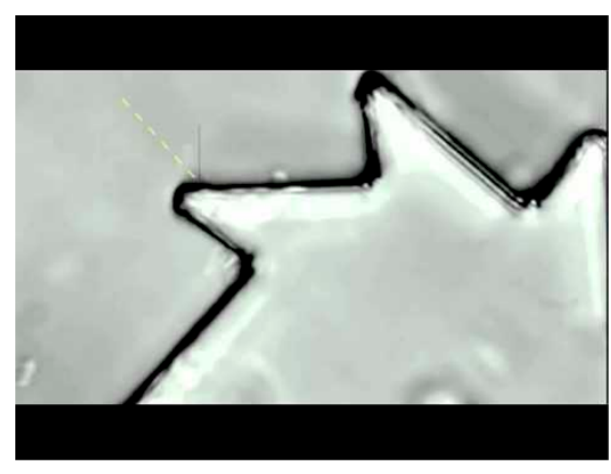

Bacteria driving a motor

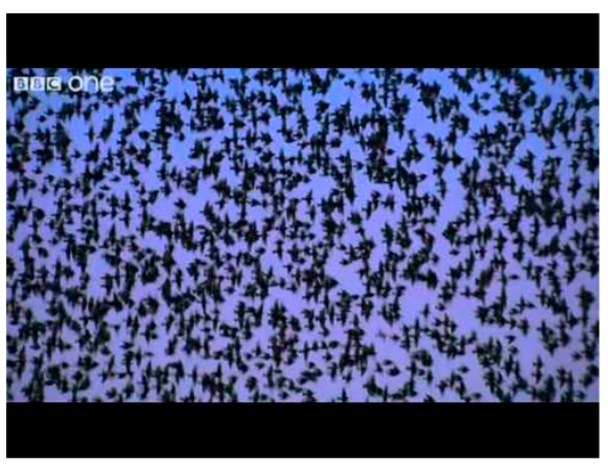

Swarming birds 


\section{A stochastic active model}

$$
\begin{gathered}
d x(t)=u(t) d t \\
m d u(t)=-\gamma u(t) d t+\sqrt{2 \gamma T_{b}} d W(t)+f_{a}(t) d t-\phi^{\prime}[x(t)] d t
\end{gathered}
$$$$
d f_{a}(t)=-\frac{f_{a}(t)}{\tau} d t+\frac{\gamma \sqrt{2 D_{a}}}{\tau} d W_{2}(t) \quad T_{a}=\gamma D_{a}
$$

$$
d x(t)=\frac{\sqrt{2 \gamma T_{b}} d W(t)+f_{a}(t) d t-\phi^{\prime}[x(t)] d t}{\gamma}
$$




\section{Heat into the solvent}

$\delta w=u f_{a} d t \quad \delta q_{b}=u \circ\left[-\gamma u d t+\sqrt{2 \gamma T_{b}} d W(t)\right]$

$$
\begin{aligned}
& \delta \sigma=d s-\frac{\delta q_{b}}{T} \\
& Q_{b}=\left\langle q_{b}\right\rangle \square \frac{\frac{\delta Q_{b}}{T} \leq 0}{{ }_{\text {Steady state }}}
\end{aligned}
$$




\section{Neglecting thermal noise}

$$
\begin{gathered}
\dot{x}=\frac{f_{a}(t)-\phi^{\prime}(x)}{\gamma} \\
d x(t)=u(t) d t
\end{gathered}
$$

$$
\begin{aligned}
\mu d u(t) & =-\gamma u(t) d t+\sqrt{2 \gamma T_{a}} d W(t)-\phi^{\prime}[x(t)] d t-\tau \phi^{\prime \prime}[x(t)] u(t) d t \\
& =-\gamma \Gamma(x) u(t) d t+\sqrt{2 \gamma \Gamma(x) \theta(x)} d W(t)-\phi^{\prime}[x(t)] d t
\end{aligned}
$$

$$
\mu=\gamma \tau \quad \Gamma(x)=1+\frac{\tau}{\gamma} \phi^{\prime \prime}(x) \quad \theta(x)=T_{a} / \Gamma(x)
$$




\section{Entropy production}

$$
\delta q_{a b}=u \circ d f_{a b} \quad \delta \sigma=d s-\frac{\delta q_{a b}}{\theta(x)}
$$

$$
d f_{a b}(t)=-\gamma \Gamma[x(t)] u(t) d t+\sqrt{2 \gamma \Gamma[x(t)] \theta(x)} d W(t)
$$

Power exchanged with the active heat-bath at temperature $\theta(x)$ 


\section{Clausius relation for active}

\section{particles}

$$
\begin{gathered}
\text { In the steady state }\left\langle\frac{\delta q_{a b}(x)}{\theta(x)}\right\rangle \leq 0 \\
\left\langle\frac{\delta q_{a b}(x)}{\theta(x)}\right\rangle=\int d x \frac{\dot{\tilde{q}}(x)}{\theta(x)} \\
\dot{\tilde{q}}(x)=\gamma \Gamma(x)\left[\frac{\theta(x)}{\mu} n(x)-\int d v u^{2} p(x, u)\right]
\end{gathered}
$$




\section{An example}

$$
w(x) \equiv \phi(x)=\frac{x^{4}}{4}-\frac{x^{2}}{2}
$$

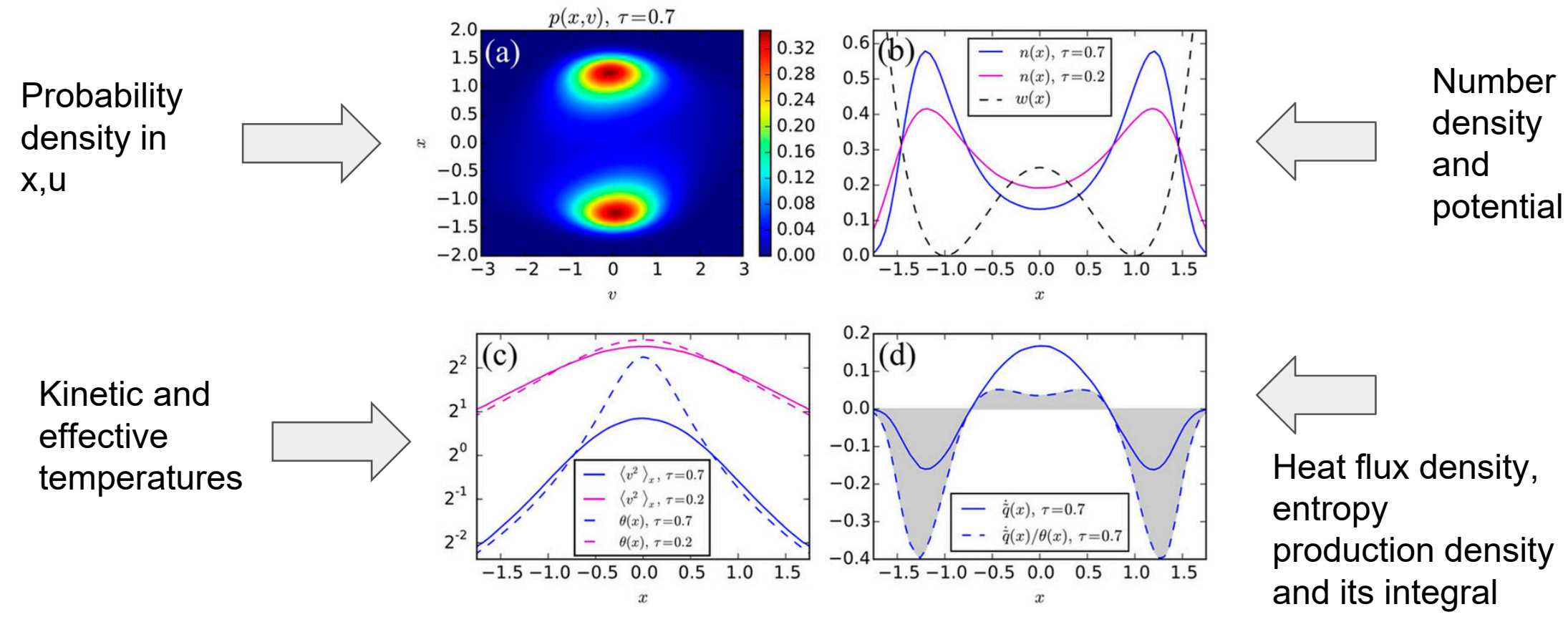




\section{In more than 1 dimension}

$$
\begin{gathered}
\mu d u_{i}=-\gamma \Gamma_{i j}(\mathbf{r}) u_{j} d t-\partial_{i} \phi(\mathbf{r}) d t+\gamma \sqrt{2 D_{a}} d W_{i} \\
\Gamma_{i j}=\delta_{i j}+\frac{\tau}{\gamma} \partial_{j} \partial_{i} \phi \\
P \Gamma P^{T}=D \quad D_{i j}(\mathbf{r})=\lambda_{i}(\mathbf{r}) \delta_{i j} \\
\delta \sigma(t)=d s(t)-\sum_{i} \frac{\delta q_{a b, i}(t)}{\theta_{i}[\mathbf{R}(t)]} \quad \theta_{i}(\mathbf{R})=T_{a} / \lambda_{i}(\mathbf{R}) \\
\delta q_{a b, i}=U_{i} \circ\left[-\gamma \lambda_{i}(\mathbf{R}) U_{i} d t+\gamma \sqrt{2 D_{a}} d W\right]
\end{gathered}
$$


Thanks for your attention! 